\title{
CHARACTERIZATION OF CALABI-YAU VARIATIONS OF HODGE STRUCTURE OVER TUBE DOMAINS BY CHARACTERISTIC FORMS
}

\author{
COLLEEN ROBLES
}

\begin{abstract}
Sheng and Zuo's characteristic forms are invariants of a variation of Hodge structure. We show that they characterize Gross's canonical variations of Hodge structure of Calabi-Yau type over (Hermitian symmetric) tube domains.
\end{abstract}

\section{INTRODUCTION}

1.1. The problem. To every tube domain $\Omega=G / K$ Gross [9] has associated a canonical (real) variation of Hodge structure (VHS)

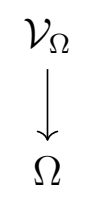

of Calabi-Yau (CY) type. The construction of (1.1) is representation theoretic, not geometric, in nature; in particular, the variation is not, a priori, induced by a family

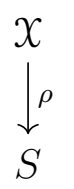

of polarized, algebraic Calabi-Yau manifolds. So an interesting problem is to construct such a family realizing (1.1). By "realize" we mean the following: let

$$
\tau: \Omega \rightarrow D_{\Omega}
$$

be the period map associated with (1.1), and $\tilde{\Phi}_{\rho}: \tilde{S} \rightarrow D$ be the (lifted) period map associated with (1.2); then we are asking for an identification $D \simeq D_{\Omega}$ with respect to which $\tilde{\Phi}_{\rho}(\tilde{S})$ is an open subset of $\tau(\Omega)$.

Date: June 2, 2017.

Robles is partially supported by NSF grants DMS 1361120 and 1611939 . 
Example 1.4. One may obtain a family of $n$-folds by resolution of double covers of $\mathbb{P}^{n}$ branched over $2 n+2$ hyperplanes in general position. When $n=1,2$, the associated VHS is a geometric realization of Gross's type $A$ canonical VHS over $\Omega=\mathrm{SU}(n, n) / \mathrm{S}(\mathrm{U}(n) \times \mathrm{U}(n))$. For $n=1$ this is the classical case of elliptic curves branched over fours points in $\mathbb{P}^{1}$. In the case $n=2$ this was proved by Matsumoto, Sasaki and Yoshida [14]. However, for $n \geq 3$, the family does not realize Gross's type $A$ canonical VHS $[4,16]$, cf. Example 1.5.

A necessary condition for (1.2) to realize (1.1) is that invariants associated to (1.1) and (1.2) agree. For example, $\operatorname{dim} S=\operatorname{dim} \Omega$, and the Hodge numbers $\mathbf{h}_{\rho}$ and $\mathbf{h}_{\Omega}$ must agree. (Of course, the latter implies that we may identify $D$ with $D_{\Omega}$.) These are discrete invariants. Sheng and Zuo's characteristic forms [17, §3] are infinitesimal, differential-geometric invariants associated with holomorphic, horizontal maps (such as $\tau$ and $\tilde{\Phi}_{\rho}$ ). In particular, the characteristic forms will necessarily agree when (1.2) realizes $(1.1)$.

Example 1.5. When $n \geq 3$ the family of Calabi-Yau's in Example 1.4 does not realize Gross's type $A$ canonical VHS over $\Omega=\mathrm{SU}(n, n) / S(\mathrm{U}(n) \times \mathrm{U}(n))$. (However, the two discrete invariants above do agree.) This was proved by Gerkmann, Sheng, van Straten and Zuo [4] in the $n=3$ case, and their argument was extended to $n \geq 3$ by Sheng, Xu and Zuo [16]. The crux of the argument is to show that the second characteristic forms do not agree. (In fact, their zero loci are not of the same dimension if $n \geq 3$. $)^{1}$

The purpose of this paper is to show that agreement of the characteristic forms is both necessary and sufficient for (1.2) to realize (1.1). We will consider a more general situation, replacing the period map $\tilde{\Phi}_{\rho}: \tilde{S} \rightarrow D \simeq D_{\Omega}$ with an arbitrary horizontal, holomorphic map $f: M \rightarrow \check{D}_{\Omega}$ into the compact dual, and asking when $f$ realizes (1.1). The first main result is stated precisely in Theorem 3.10. To state the informal version, we first recall that Gross's canonical VHS is given by a real

\footnotetext{
${ }^{1} \mathrm{~A}$ similar argument was used by Sasaki, Yamaguchi and Yoshida [15] to disprove a related conjecture on the projective solution of the system of hypergeometric equations associated with the hyperplane configurations.
} 
representation

$$
G \rightarrow \operatorname{Aut}(U, Q):=\{g \in \operatorname{Aut}(U) \mid Q(g u, g v)=Q(u, v), \forall u, v \in U\}
$$

the period domain $D_{\Omega}$ parameterizes (real) $Q$-polarized Hodge structures on $U$ of Calabi-Yau type; and the period map (1.3) extends to a $G_{\mathbb{C}^{-}}$equivariant map $\tau$ : $\check{\Omega} \rightarrow \check{D}_{\Omega}$ between the compact duals.

Main Theorem 1 (Informal statement of Theorem 3.10). If the characteristic forms of $f$ and $\tau$ are isomorphic, then there exists $g \in \operatorname{Aut}\left(U_{\mathbb{C}}\right)$ so that $g \circ f(M)$ is an open subset of $\tau(\check{\Omega})$.

Characteristic forms are defined in $\S 2$. The statement of Theorem 3.10 is a bit stronger than the above: in fact, it suffices to check that the characteristic forms of $f$ are isomorphic to those of $\tau$ at a single point $x \in M$, so long as the integer-valued differential invariants (§2.3) associated with $f$ are constant in a neighborhood of $x$. Theorem 3.10 is a consequence of: (i) an identification of the characteristic forms of Gross's (1.1) with the fundamental forms of the minimal homogeneous embedding $\sigma: \check{\Omega} \hookrightarrow \mathbb{P} U_{\mathbb{C}}$ (Proposition 4.4), and (ii) Hwang and Yamaguchi's characterization [10] of compact Hermitian symmetric spaces by their fundamental forms.

Main Theorem 1 characterizes horizontal maps realizing Gross's canonical VHS modulo the full linear automorphism group $\operatorname{Aut}\left(U_{\mathbb{C}}\right)$. It is natural to ask if we can characterize the horizontal maps realizing Gross's VHS up to the (smaller) group $\operatorname{Aut}\left(U_{\mathbb{C}}, Q\right)$ preserving the polarization — these groups are the natural symmetry groups of Hodge theory. (Note that $\operatorname{Aut}\left(U_{\mathbb{C}}, Q\right)$ is the automorphism group of $\check{D}_{\Omega}$, the full $\operatorname{Aut}\left(U_{\mathbb{C}}\right)$ does not preserve the compact dual.) The second main result does exactly this. This congruence requires a more refined notion of agreement of the characteristic forms than the isomorphism of Main Theorem 1; the precise statement is given in Theorem 5.14. The refinement is encoded by the condition that a certain vector-valued differential form $\eta$ vanishes on a frame bundle $\mathcal{E}_{f} \rightarrow M$ (cf. Remark 5.21(b)). Informally, one begins with a frame bundle $\mathcal{E}_{Q} \rightarrow \check{D}_{\Omega}$ with fibre over $\left(F^{p}\right) \in \check{D}_{\Omega}$ consisting of all bases $\left\{e_{0}, \ldots, e_{d}\right\}$ of $U_{\mathbb{C}}$ such that $Q\left(e_{j}, e_{k}\right)=\delta_{j+k}^{d}$ and $F^{p}=\operatorname{span}\left\{e_{0}, \ldots, e_{d^{p}}\right\}$. The bundle $\mathcal{E}_{Q}$ is isomorphic to the Lie group $\operatorname{Aut}\left(U_{\mathbb{C}}, Q\right)$, and so inherits the left-invariant, Maurer-Cartan form $\theta$ which takes values in the Lie 
algebra

$$
\operatorname{End}\left(U_{\mathbb{C}}, Q\right):=\left\{X \in \operatorname{End}\left(U_{\mathbb{C}}\right) \mid Q(X u, v)+Q(u, X v)=0, \forall u, v \in U_{\mathbb{C}}\right\}
$$

of $\operatorname{Aut}\left(U_{\mathbb{C}}, Q\right)$. There is a $G_{\mathbb{C}^{-}}$module decomposition $\operatorname{End}\left(U_{\mathbb{C}}, Q\right)=\mathfrak{g}_{\mathbb{C}} \oplus \mathfrak{g}_{-}^{\perp} \oplus \mathfrak{g}_{\geq 0}^{\perp}$; let $\eta=\theta_{\mathfrak{g}_{\perp}^{\perp}}$ be the component of $\theta$ taking value in $\mathfrak{g}_{-}^{\perp}$.

Main Theorem 2 (Informal statement of Theorem 5.14). Let $f: M \rightarrow \check{D}_{\Omega}$ be a holomorphic, horizontal map. There exists $g \in \operatorname{Aut}\left(U_{\mathbb{C}}, Q\right)$ so that $g \circ f(M)$ is an open subset of $\tau(\check{\Omega}) \subset \check{D}_{\Omega}$ if and only if $\eta$ vanishes on the pull-back $\mathcal{E}_{f}:=f^{*} \mathcal{E}_{Q} \rightarrow M$.

Roughly speaking, $\eta$ vanishes on $\mathcal{E}_{f}$ if and only if the coefficients of the fundamental forms of $f$ agree with those of Gross's canonical CY-VHS when expressed in terms of bases $\mathbf{e} \in \mathcal{E}_{Q}$ (Remark 5.21). Main Theorem 2 is reminiscent of Green-GriffithsKerr's characterization of nondegenerate complex variations of quintic mirror Hodge structures by the Yukawa coupling (another differential invariant associated to a VHS) [5, §IV]. Both Main Theorems 1 and 2, and the Green-Griffiths-Kerr characterization, are solutions to equivalence problems in the sense of É. Cartan. And from that point of view, the formulation of Main Theorem 2 is standard in that it characterizes equivalence by the vanishing of a certain form on a frame bundle over $M$.

The proof of Theorem 5.14 is established by a minor modification of the arguments employed in [13] (which are similar to those of [10]), and is in the spirit of Cartan's approach to equivalence problems via the method of moving frames.

Remark 1.7. Mao and Sheng [17, 2 ] extended Gross's construction of the canonical real CY-VHS over a tube domain to a canonical complex CY-VHS over a bounded symmetric domain. The analogs of Theorems 3.10 and 5.14 hold for the Mao-Sheng CY-VHS as well. Specifically, the definition of the characteristic forms holds for arbitrary (not necessarily real) VHS; and the arguments establishing the theorems do not make use of the hypotheses that the bounded symmetric domain $\Omega$ is of tube type or that the VHS is real. As indicated by the proofs of Theorems 3.10 and 5.14, the point at which some care must be taken is when considering the case that $\check{\Omega}$ is either a projective space or a quadric hypersurface. If $\check{\Omega}$ is not of tube type, then it can not be a quadric hypersurface. If $\check{\Omega}$ is a projective space, then $\check{\Omega}=\check{D}_{\Omega}$, and the theorems are trivial. 
1.2. Notation. Throughout $V$ will denote a real vector space, and $V_{\mathbb{C}}$ the complexification. All Hodge structures are assumed to be effective; that is, the Hodge numbers $h^{p, q}$ vanish if either $p$ or $q$ is negative. Throughout $\check{D}$ will denote the compact dual of a period domain $D$ parameterizing effective, polarized Hodge structures of weight $n$ on $V$. Here $D$ and $V$ are arbitrary; we will reserve $D_{\Omega}$ and $U$ for the period domain and vector space specific to Gross's canonical variation of Hodge structure. We will let $Q$ denote the polarization on both $V$ and $U$, as which is meant will be clear from context.

\section{Characteristic Forms}

\subsection{Horizontality. Let}

$$
\mathcal{F}^{n} \subset \mathcal{F}^{n-1} \subset \cdots \subset \mathcal{F}^{1} \subset \mathcal{F}^{0}
$$

denote the canonical filtration of the trivial bundle $\mathcal{F}^{0}=\check{D} \times V_{\mathbb{C}}$ over $\check{D}$. Given a holomorphic map $f: M \rightarrow \check{D}$, let

$$
\mathcal{F}_{f}^{p}:=f^{*} \mathcal{F}^{p}
$$

denote the pull-back of the Hodge bundles to $M$. We say that $f$ is horizontal if it satisfies the infinitesimal period relation (IPR)

$$
\mathrm{d} \mathcal{F}_{f}^{p} \subset \mathcal{F}_{f}^{p-1} \otimes \Omega_{M}^{1} .
$$

Example 2.3. The lifted period map $\tilde{\Phi}: \tilde{S} \rightarrow D$ arising from a family $X \rightarrow S$ of polarized, algebraic manifolds is a horizontal, holomorphic map [7, 8].

2.2. Definition. Given a horizontal map $f: M \rightarrow \check{D}$, the IPR (2.2) yields a vector bundle map

$$
\gamma_{f}: T M \rightarrow \operatorname{Hom}\left(\mathcal{F}_{f}^{n}, \mathcal{F}_{f}^{n-1} / \mathcal{F}_{f}^{n}\right) ;
$$

sending $\xi \in T_{x} M$ to the linear map $\gamma_{f, x}(\xi) \in \operatorname{Hom}\left(\mathcal{F}_{f, x}^{n}, \mathcal{F}_{f, x}^{n-1} / \mathcal{F}_{f, x}^{n}\right)$ defined as follows. Fix a locally defined holomorphic vector field $X$ on $M$ extending $\xi=X_{x}$. Given any $v_{0} \in \mathcal{F}_{f, x}^{n}$, let $v$ be a local section of $\mathcal{F}_{f}^{n}$ defined in a neighborhood of $x$ and with $v(x)=v_{0}$. Then

$$
\gamma_{f}(\xi)\left(v_{0}\right):=\left.X(v)\right|_{x} \bmod \mathcal{F}_{f, x}^{n}
$$


yields a well-defined map $\gamma_{f}(\xi) \in \operatorname{Hom}\left(\mathcal{F}_{f}^{n}, \mathcal{F}_{f}^{n-1} / \mathcal{F}_{f}^{n}\right)$. More generally there is a vector bundle map

$$
\gamma_{f}^{k}: \operatorname{Sym}^{k} T M \rightarrow \operatorname{Hom}\left(\mathcal{F}_{f}^{n}, \mathcal{F}_{f}^{n-k} / \mathcal{F}_{f}^{n-k+1}\right)
$$

defined as follows. Given $\xi_{1}, \ldots, \xi_{k} \in T_{x} M$, let $X_{1}, \ldots, X_{k}$ be locally defined holomorphic vector fields extending the $\xi_{j}=X_{j, x}$. Given $v_{0}$ and $v$ as above, define

$$
\gamma_{f}^{k}\left(\xi_{1}, \ldots, \xi_{k}\right)\left(v_{0}\right):=\left.X_{1} \cdots X_{k}(v)\right|_{x} \bmod \mathcal{F}_{f, x}^{n-k+1}
$$

It is straightforward to confirm that $\gamma_{f}^{k}$ is well-defined. This bundle map is the $k$-th characteristic form of $f: M \rightarrow \check{D}$. Let $\mathbf{C}_{f}^{k} \subset \operatorname{Sym}^{k} T^{*} M$ denote the image of the dual map. In a mild abuse of terminology we will also call $\mathbf{C}_{f}^{k}$ the $k$-th characteristic forms of $f: M \rightarrow \check{D}$.

2.3. Isomorphism. Given two horizontal maps $f: M \rightarrow \check{D}$ and $f^{\prime}: M^{\prime} \rightarrow \check{D}$, we say that the characteristic forms of $f$ at $x$ are isomorphic to those of $f^{\prime}$ at $x^{\prime}$ if there exists a linear isomorphism $\lambda: T_{x} M \rightarrow T_{x^{\prime}} M^{\prime}$ such that the induced linear map $\lambda^{k}: \operatorname{Sym}^{k}\left(T_{x^{\prime}}^{*} M^{\prime}\right) \rightarrow \operatorname{Sym}^{k}\left(T_{x}^{*} M\right)$ identifies $\mathbf{C}_{f^{\prime}, x^{\prime}}^{k}$ with $\mathbf{C}_{f, x}^{k}$, for all $k \geq 0$.

Each $\mathbf{C}_{f, x}^{k}$ is a vector subspace of $\operatorname{Sym}^{k} T_{x}^{*} M$, and

$$
c_{f, x}^{k}:=\operatorname{dim}_{\mathbb{C}} \mathbf{C}_{f, x}^{k} \leq \operatorname{dim} \mathcal{F}_{f, x}^{n-k} / \mathcal{F}_{f, x}^{n-k+1}
$$

is an example of an "integer-valued differential invariant of $f: M \rightarrow \check{D}$ at $x$." Let

$$
\mathbf{C}_{f, x}:=\bigoplus_{k \geq 0} \mathbf{C}_{f, x}^{k} \subset \bigoplus_{k \geq 0} \operatorname{Sym}^{k} T_{x}^{*} M=: \operatorname{Sym}_{x}^{*} M
$$

and set $c_{f, x}:=\operatorname{dim}_{\mathbb{C}} \mathbf{C}_{f, x}=\sum_{k>0} c_{f, x}^{k}$. Regard $\mathbf{C}_{f, x}$ as an element of the Grassmannian $\operatorname{Gr}\left(c_{f, x}, \operatorname{Sym} T_{x}^{*} M\right)$. Note that $\operatorname{Aut}\left(T_{x} M\right)$ acts on this Grassmannian. By integer-valued differential invariant of $f: M \rightarrow \check{D}$ at $x$ we mean the value at $\mathbf{C}_{f, x}$ of any $\operatorname{Aut}\left(T_{x} M\right)$-invariant integer-valued function on $\operatorname{Gr}\left(c_{f, x}, \operatorname{Sym} T_{x}^{*} M\right)$.

A necessary condition for two characteristic forms $\mathbf{C}_{f, x}$ and $\mathbf{C}_{f^{\prime}, x^{\prime}}$ to be isomorphic is that the integer-valued differential invariants at $x$ and $x^{\prime}$, respectively, agree. 


\section{Gross's CAnOnical CY-VHS}

3.1. Maps of Calabi-Yau type. A period domain $D$ parameterizing effective polarized Hodge structures of weight $n$ is of Calabi-Yau type (CY) if $h^{n, 0}=1$. In this case we also say that the compact dual $\check{D}$ is of Calabi-Yau type.

A holomorphic, horizontal map $f: M \rightarrow \check{D}$ is of Calabi-Yau (CY) type if $\check{D}$ is $\mathrm{CY}$ and $\gamma_{f, x}: T_{x} M \rightarrow \operatorname{Hom}\left(\mathcal{F}_{f, x}^{n}, \mathcal{F}_{f, x}^{n-1} / \mathcal{F}_{f, x}^{n}\right)$ is a linear isomorphism for all $x \in M$.

Remark 3.1. In particular, if $f: M \rightarrow \check{D}$ and $f^{\prime}: M^{\prime} \rightarrow \check{D}$ are CY, then the first characteristic forms $\mathbf{C}_{f, x}^{1}$ and $\mathbf{C}_{f^{\prime}, x^{\prime}}^{1}$ are always isomorphic, for any $x \in M$ and $x^{\prime} \in M^{\prime}$.

The condition that $h^{n, 0}=\operatorname{rank}_{\mathbb{C}} \mathcal{F}^{n}=1$ implies that there is an map

$$
\pi: \check{D} \rightarrow \mathbb{P} V_{\mathbb{C}}
$$

sending $\phi \in D$ to $\mathcal{F}_{\phi}^{n} \in \mathbb{P} V_{\mathbb{C}}$.

3.2. Definition. We briefly recall Gross's canonical CY-VHS over a tube domain $\Omega=G / K[9]$. Up to $G$-module isomorphism, there is a unique real representation

$$
G \rightarrow \operatorname{Aut}(U)
$$

with the following properties:

(i) The complexification $U_{\mathbb{C}}$ is an irreducible $G$-module.

(ii) The maximal compact subgroup $K \subset G$ is the stabilizer of a highest weight line $\ell \subset U_{\mathbb{C}}$. In particular, if $P \subset G_{\mathbb{C}}$ is the stabilizer of $\ell$, then $K=G \cap P$, and the map $g P \mapsto g \cdot \ell \in \mathbb{P} U_{\mathbb{C}}$ is a $G_{\mathbb{C}}-$ equivariant homogeneous embedding

$$
\sigma: \check{\Omega} \hookrightarrow \mathbb{P} U_{\mathbb{C}}
$$

of the compact dual $\check{\Omega}=G_{\mathbb{C}} / P$ of $\Omega$.

(iii) The dimension of $U$ is minimal amongst all $G$-modules with the two properties above.

The maximal compact subgroup $K$ is the centralizer of a circle $\varphi: S^{1} \rightarrow G$ (a homomorphism of $\mathbb{R}$-algebraic groups). The representation $U_{\mathbb{C}}$ decomposes as a direct 
sum

$$
U_{\mathbb{C}}=\bigoplus_{p+q=n} U^{p, q}
$$

of $\varphi$-eigenspaces

$$
U^{p, q}:=\left\{u \in U_{\mathbb{C}} \mid \varphi(z) u=z^{p-q} u\right\}
$$

This is a Hodge decomposition, and there exists a $G$-invariant polarization $Q$ of the Hodge structure; in particular, the representation (3.2) takes values in $\operatorname{Aut}(U, Q)$ :

$$
G \rightarrow \operatorname{Aut}(U, Q) .^{2}
$$

Each subset $U^{p, q}$ is $K$-invariant, and so defines a $G$-homogeneous bundle $\mathcal{U}^{p, q}$ over $\Omega$. The resulting decomposition

$$
\Omega \times U_{\mathbb{C}}=\bigoplus \mathcal{U}^{p, q}
$$

of the trivial bundle over $\Omega$ is Gross's canonical VHS over $\Omega$ [9].

Example 3.7. In the case that $\Omega$ is irreducible, Gross's canonical CY-VHS is one of the following six:

(a) For $G=\mathrm{U}(n, n)=\operatorname{Aut}\left(\mathbb{C}^{2 n}, \mathcal{H}\right)$, we have $U_{\mathbb{C}}=\bigwedge^{n} \mathbb{C}^{2 n}$ and $\check{\Omega}=\operatorname{Gr}\left(n, \mathbb{C}^{2 n}\right)$. If $\mathbb{C}^{2 n}=A \oplus B$ is the $\varphi$-eigenspace decomposition, then $n=\operatorname{dim} A=\operatorname{dim} B$ and the Hermitian form $\mathcal{H}$ restricts to a definite form on both $A$ and $B$. The Hodge decomposition is given by $U^{p, q} \simeq\left(\bigwedge^{p} A\right) \otimes\left(\bigwedge^{q} B\right)$.

(b) For $G=\mathrm{O}(2, k)=\operatorname{Aut}\left(\mathbb{R}^{2+k}, Q\right)$, we have $U_{\mathbb{C}}=\mathbb{C}^{2+k}$ and $\Omega$ is the period domain parameterizing $Q$-polarized Hodge structures on $U=\mathbb{R}^{2+k}$ with $\mathbf{h}=(1, k, 1)$, so that $\check{\Omega}$ is the quadric hypersurface $\{Q=0\} \subset \mathbb{P}^{k+1}$.

(c) For $G=\operatorname{Sp}(2 g, \mathbb{R})=\operatorname{Aut}\left(\mathbb{R}^{2 g}, Q\right)$, we have $U_{\mathbb{C}}=\bigwedge^{g} \mathbb{C}^{2 g}$ and $\Omega$ is the period domain parameterizing $Q$-polarized Hodge structures on $\mathbb{C}^{2 g}$ with $\mathbf{h}=(g, g)$, so that $\check{\Omega}$ is the Lagrangian grassmannian of $Q$-isotropic $g$-planes in $\mathbb{C}^{2 g}$. Given one such Hodge decomposition $\mathbb{C}^{2 n}=A \oplus B$, the corresponding Hodge structure on $U$ is given by $U^{p, q}=\left(\bigwedge^{p} A\right) \oplus\left(\bigwedge^{q} B\right)$.

\footnotetext{
${ }^{2}$ In the terminology of [6], the triple $\left(U_{\mathbb{C}}, \varphi, Q\right)$ defines a Hodge representation which realizes the tube domain $\Omega$ as a Mumford-Tate domain.
} 
(d) For $G=\mathrm{SO}^{*}(2 n), U_{\mathbb{C}}$ is a Spinor representation, and the summands of the Hodge decomposition are $U^{p, q} \simeq \bigwedge^{2 p} \mathbb{C}^{2 n}$.

(e) If $G$ is the exceptional simple real Lie group of rank 7 with maximal compact subgroup $K=U(1) \times_{\mu_{3}} E_{6}$, then the Hodge decomposition is $U_{\mathbb{C}} \simeq \mathbb{C} \oplus \mathbb{C}^{27} \oplus$ $\left(\mathbb{C}^{27}\right)^{*} \oplus \mathbb{C}$.

Lemma 3.8 (Gross [9]). Gross's canonical VHS (3.6) is of Calabi-Yau type (§3.1).

The lemma follows from the well-understood representation theory associated with (3.3) and (3.5). We briefly review the argument below as a means of recalling those representation theoretic properties that will later be useful. (See [9] for details.)

Let

$$
\varphi \in D_{\Omega}
$$

denote the Hodge structure given by (3.4). The map

$$
\tau: \check{\Omega} \hookrightarrow \check{D}_{\Omega}
$$

sending $g P \mapsto g \cdot \varphi$ is a $G_{\mathbb{C}^{-}}$equivariant homogeneous embedding of the compact dual $\check{\Omega}=G_{\mathbb{C}} / P$. The restriction of $\tau$ to $\Omega$ is the period map associated to Gross's canonical CY-VHS. The precise statement of Main Theorem 1 is

Theorem 3.10. Let $f: M \hookrightarrow \check{D}_{\Omega}$ be any $C Y$ map (§3.1), and let $x \in M$ be a point admitting a neighborhood in which all integer-valued differential invariants of $f$ are constant (\$2.3). If the characteristic forms of $f$ at $x$ are isomorphic to the characteristic forms of $\tau: \check{\Omega} \hookrightarrow \check{D}_{\Omega}$ at $o \in \Omega$ in the sense of $\S 2.3$, then there exists $g \in \operatorname{Aut}\left(U_{\mathbb{C}}\right)$ so that $g \circ f(M)$ is an open subset of $\tau(\check{\Omega})$.

The theorem is proved in $\S 4.4$.

Remark 3.11. To see how Main Theorem 1 follows from Theorem 3.10 we make precise the hypothesis that "the characteristic forms of $f$ and $\tau$ are isomorphic": by this, we mean that there exists a local biholomorphism $i: M \rightarrow \check{\Omega}$ so that the characteristic forms of $f$ at $x \in M$ are isomorphic to those of $\tau$ at $i(x)$ for all $x \in M$ (cf. §2.3). (Equivalently, since $\check{\Omega}$ is homogeneous, the characteristic forms of $f$ at $x \in M$ are isomorphic to those of $\tau$ at $o$ for all $x \in M$.) Given this definition, it is clear that the hypotheses of Main Theorem 1 imply those of Theorem 3.10. 
Proof of Lemma 3.8. Let

$$
\mathbf{h}_{\Omega}=\left(h_{\Omega}^{p, q}=\operatorname{dim}_{\mathbb{C}} U^{p, q}\right)
$$

denote the Hodge numbers, and let $D_{\Omega}$ denote the period domain parameterizing $Q$-polarized Hodge structures on $U$ with Hodge numbers $\mathbf{h}_{\Omega}$. The weight $n$ of the Hodge structure is the rank of $\Omega$, and the highest weight line stabilized by $K$ is

$$
\ell=U^{n, 0} .
$$

In particular,

$$
h^{n, 0}=1 .
$$

Let

$$
0 \subset \mathcal{F}_{\Omega}^{n} \subset \mathcal{F}_{\Omega}^{n-1} \subset \cdots \subset \mathcal{F}_{\Omega}^{1} \subset \mathcal{F}_{\Omega}^{0}
$$

denote the canonical filtration (2.1) of the trivial bundle $\mathcal{F}_{\Omega}^{0}=\check{D}_{\Omega} \times U_{\mathbb{C}}$ over $\check{D}_{\Omega}$. Then

$$
\left.\mathcal{F}_{\Omega}^{p}\right|_{\tau(\Omega)}=\bigoplus_{r \geq p} \mathcal{U}^{r, n-r}
$$

We will identify

$$
o=K / K \in \Omega=G / K
$$

with $P / P \in \check{\Omega}=G_{\mathbb{C}} / P$. Note that

$$
\varphi=\tau(o)
$$

The weight zero Hodge decomposition

$$
\mathfrak{g}_{\mathbb{C}}=\mathfrak{g}_{\varphi}^{1,-1} \oplus \mathfrak{g}_{\varphi}^{0,0} \oplus \mathfrak{g}_{\varphi}^{-1,1}
$$

induced by $\varphi$ has the property that $\mathfrak{p}=\mathfrak{g}_{\varphi}^{1,-1} \oplus \mathfrak{g}_{\varphi}^{0,0}$ and $\mathfrak{k}_{\mathbb{C}}=\mathfrak{g}_{\varphi}^{0,0}$ are the Lie algebras of $P$ and $K_{\mathbb{C}}$, respectively. Consequently, the holomorphic tangent space is given by

$$
T_{o} \Omega=T_{o} \check{\Omega}=\mathfrak{g}_{\mathbb{C}} / \mathfrak{p} \simeq \mathfrak{g}_{\varphi}^{-1,1} .
$$

Regarding $\mathfrak{g}_{\varphi}^{-1,1}$ as a subspace of $\operatorname{End}\left(U_{\mathbb{C}}, Q\right)$ we have

$$
U^{p-1, q+1}=\mathfrak{g}_{\varphi}^{-1,1}\left(U^{p, q}\right):=\left\{\xi(u) \mid \xi \in \mathfrak{g}_{\varphi}^{-1,1}, u \in U^{p, q}\right\} .
$$

In particular, given $\xi \in \mathfrak{g}_{\varphi}^{-1,1}$, we have

$$
\xi\left(U^{p, q}\right) \subset U^{p-1, q+1} .
$$


The maps

$$
\psi_{\Omega}^{p, q}: \mathfrak{g}_{\varphi}^{-1,1} \times U^{p, q} \rightarrow U^{p-1, q+1}
$$

sending

$$
(\xi, u) \mapsto \xi(u)
$$

are surjective. Moreover, given fixed nonzero $u_{0} \in U^{n, 0}$, the map $\mathfrak{g}_{\varphi}^{-1,1} \rightarrow U^{n-1,1}$ sending $\xi \mapsto \xi\left(u_{0}\right)$ is an isomorphism. It follows from the homogeneity of the bundles $\mathcal{F}_{\Omega}^{p}$, and the $G_{\mathbb{C}}$-equivariance of $\tau$, that $\tau$ is horizontal and of Calabi-Yau type.

3.3. Characteristic forms. In this section we describe the characteristic forms $\gamma_{\Omega}^{k}$ of (3.9). The discussion will make use of results reviewed in the proof of Lemma 3.8.

Since $\tau$ is $G_{\mathbb{C}^{-}}$equivariant and the bundles $\mathcal{F}_{\Omega}^{p} \rightarrow \check{D}_{\Omega}$ are $\operatorname{Aut}\left(U_{\mathbb{C}}, Q\right)$-homogeneous, we see that the push-forward $g_{*}: T_{o} \check{\Omega} \rightarrow T_{g \cdot o} \check{\Omega}$ is an isomorphism identifying $\mathbf{C}_{\tau, g \cdot o}^{k}$ with $\mathbf{C}_{\tau, o}^{k}$ for all $k$ and $g \in G_{\mathbb{C}}$; that is, the characteristic forms of $\tau$ at $g \cdot o$ are isomorphic to those at $o$. So it suffices to describe the characteristic forms at the point $o \in \Omega$. It follows from $\mathcal{F}_{\Omega, o}^{p} / \mathcal{F}_{\Omega, o}^{p+1}=U^{p, n-p}$, the identification (3.15), and (3.17) that $\gamma_{\Omega, o}^{k}: \operatorname{Sym}^{k} T_{o} \check{\Omega} \rightarrow \operatorname{Hom}\left(\mathcal{F}_{\Omega, o}^{n}, \mathcal{F}_{\Omega, o}^{n-k} / \mathcal{F}_{\Omega, o}^{n-k+1}\right)$ may be identified with the map

$$
\gamma_{\Omega, o}^{k}: \operatorname{Sym}^{k} \mathfrak{g}_{\varphi}^{-1,1} \rightarrow \operatorname{Hom}\left(U^{n, 0}, U^{n-k, k}\right)
$$

defined by

$$
\gamma_{\Omega, o}^{k}\left(\xi_{1} \cdots \xi_{k}\right)(u)=\xi_{1} \cdots \xi_{k}(u)
$$

with $\xi_{1}, \ldots, \xi_{k} \in \mathfrak{g}_{\varphi}^{-1,1} \subset \operatorname{End}\left(U_{\mathbb{C}}, Q\right)$ and $u \in U^{n, 0}$.

\section{Proof of Theorem 3.10 (Main Theorem 1)}

4.1. The osculating filtration. Let $X \hookrightarrow \mathbb{P} V_{\mathbb{C}}$ be any complex submanifold. The osculating filtration at $x \in X$

$$
\mathcal{T}_{x}^{0} \subset \mathcal{T}_{x}^{1} \subset \cdots \subset \mathcal{T}_{x}^{m} \subset V_{\mathbb{C}}
$$

is defined as follows. First, $\mathcal{T}_{x}^{0} \subset V_{\mathbb{C}}$ is the line parameterized by $x \in \mathbb{P} V_{\mathbb{C}}$. Let $\widehat{X} \subset V_{\mathbb{C}} \backslash\{0\}$ be the cone over $X$. Let $\Delta=\{z \in \mathbb{C}:|z|<1\}$ denote the unit disc, 
and let $\mathcal{O}(\Delta, 0 ; \widehat{X}, x)$ denote the set of holomorphic maps $\alpha: \Delta \rightarrow \widehat{X}$ with $\alpha(0) \in \mathcal{T}_{x}^{0}$. Given one such curve, let $\alpha^{(k)}$ denote the $k$-th derivative $\mathrm{d}^{k} \alpha / \mathrm{d} z^{k}$. Inductively,

$$
\mathcal{T}_{x}^{k}=\mathcal{T}_{x}^{k-1}+\operatorname{span}_{\mathbb{C}}\left\{\alpha^{(k)}(0) \mid \alpha \in \mathcal{O}(\Delta, 0 ; X, x)\right\}
$$

Note that $\mathcal{T}_{x}^{1}=T_{u} \widehat{X}$ is the embedded tangent space at $u \in \mathcal{T}_{x}^{0}$. Here $m=m(x)$ is determined by $\mathcal{T}_{x}^{m-1} \subsetneq \mathcal{T}_{x}^{m}=\mathcal{T}_{x}^{m+1}$.

4.2. Fundamental forms. If both $m$ and the rank of $\mathcal{T}_{x}^{k}$ are independent of $x$, then the osculating filtrations define a a filtration $\mathcal{T}_{X}^{0} \subset \mathcal{T}_{X}^{1} \subset \cdots \subset \mathcal{T}_{X}^{m} \subset X \times V_{\mathbb{C}}$ of the trivial bundle over $X$. Assume this is the case. By construction the osculating filtration satisfies

$$
\mathrm{d} \mathcal{T}^{k} \subset \mathcal{T}^{k+1} \otimes \Omega_{X}^{1}
$$

Just as the IPR (2.2) lead to the characteristic forms (2.4), the relation (4.1) yields bundle maps

$$
\psi_{X}^{k}: \operatorname{Sym}^{k} T X \rightarrow \operatorname{Hom}\left(\mathcal{T}_{X}^{0}, \mathcal{T}_{X}^{k} / \mathcal{T}_{X}^{k-1}\right), \quad k \geq 1
$$

This is the $k$-th fundamental form of $X \hookrightarrow \mathbb{P} V_{\mathbb{C}}$. The image $\mathbf{F}_{X}^{k} \subset \operatorname{Sym}^{k} T^{*} X$ of the dual map is a vector subbundle of

$$
\operatorname{rank} \mathbf{F}_{X}^{k}=\operatorname{dim} \mathcal{T}_{x}^{k} / \mathcal{T}_{x}^{k-1}
$$

Again, in mild abuse of terminology, we will call $\mathbf{F}_{X}^{k}$ the $k$-th fundamental forms of $X \subset \mathbb{P} V_{\mathbb{C}}$.

Given two complex submanifolds $X, X^{\prime} \hookrightarrow \mathbb{P} V_{\mathbb{C}}$, we say that the fundamental forms of $X$ at $x$ are isomorphic to those of $X^{\prime}$ at $x^{\prime}$ if there exists a linear isomorphism $\lambda: T_{x} X \rightarrow T_{x^{\prime}} X^{\prime}$ such that the induced linear map Sym $T_{x^{\prime}}^{*} X^{\prime} \rightarrow \operatorname{Sym} T_{x}^{*} X$ identifies $\mathbf{F}_{X^{\prime}, x^{\prime}}^{k}$ with $\mathbf{F}_{X, x}^{k}$.

Each $\mathbf{F}_{X, x}^{k}$ is a vector subspace of $\operatorname{Sym}^{k} T_{x}^{*} X$, and $d_{X, x}^{k}:=\operatorname{dim}_{\mathbb{C}} \mathbf{F}_{X, x}^{k}$ is an example of an "integer-valued differential invariant of $X \hookrightarrow \mathbb{P} V_{\mathbb{C}}$ at $x$." Let

$$
\mathbf{F}_{X, x}:=\bigoplus_{k \geq 0} \mathbf{F}_{X, x}^{k} \subset \bigoplus_{k \geq 0} \operatorname{Sym}^{k} T_{x}^{*} X=: \operatorname{Sym}_{x}^{*} X
$$

and set $d_{X, x}:=\operatorname{dim}_{\mathbb{C}} \mathbf{F}_{X, x}=\sum_{k \geq 0} d_{X, x}^{k}$. Regard $\mathbf{F}_{X, x}$ as an element of the Grassmannian $\operatorname{Gr}\left(d_{X, x}, \operatorname{Sym} T_{x}^{*} X\right)$. Note that $\operatorname{Aut}\left(T_{x} X\right)$ acts on this Grassmannian. By 
integer-valued differential invariant of $X \hookrightarrow \mathbb{P} V_{\mathbb{C}}$ at $x$ we mean the value at $\mathbf{F}_{X, x}$ of any $\operatorname{Aut}\left(T_{x} X\right)$-invariant integer-valued function on $\operatorname{Gr}\left(d_{X, x}, \operatorname{Sym} T_{x}^{*} X\right)$.

A necessary condition for two fundamental forms $\mathbf{F}_{X, x}$ and $\mathbf{F}_{X^{\prime}, x^{\prime}}$ to be isomorphic is that the integer-valued differential invariants at $x$ and $x^{\prime}$, respectively, agree.

Remark 4.2. When $X \hookrightarrow \mathbb{P} V_{\mathbb{C}}$ is a homogeneous embedding of a compact Hermitian symmetric space (such as the $\sigma: \check{\Omega} \hookrightarrow \mathbb{P} U_{\mathbb{C}}$ of (3.3)), there are only finitely many $\operatorname{Aut}\left(T_{o} \check{\Omega}\right)$-invariant integer-valued functions on $\operatorname{Gr}\left(d_{\sigma, o}, \operatorname{Sym} T_{o}^{*} \check{\Omega}\right)$, and they distinguish/characterize the $\operatorname{Aut}\left(T_{o} \check{\Omega}\right)$-orbits [10, Proposition 5].

4.3. Fundamental forms for $\sigma: \check{\Omega} \hookrightarrow \mathbb{P} U_{\mathbb{C}}$. Recall the maps $\sigma$ and $\tau$ of (3.3) and (3.9), respectively. Theorem 4.3 asserts that the Hermitian symmetric $\sigma(\check{\Omega}) \subset \mathbb{P} U_{\mathbb{C}}$ are characterized by their fundamental forms, up to the action of $\operatorname{Aut}\left(U_{\mathbb{C}}\right)$.

Theorem 4.3 (Hwang-Yamaguchi [10]). Assume that the compact dual $\check{\Omega}$ contains neither a projective space nor a quadric hypersurface as an irreducible factor. Let $M \subset \mathbb{P} U_{\mathbb{C}}$ be any complex manifold, and let $x \in M$ be a point in a neighborhood of which all integer-valued differential invariants are constant. If the fundamental forms of $M$ at $x$ are isomorphic to the fundamental forms of $\sigma: \check{\Omega} \hookrightarrow \mathbb{P} U_{\mathbb{C}}$ at $o$, then $M$ is

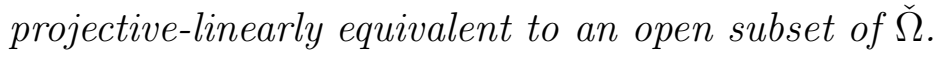

Proposition 4.4. The $k$-th characteristic form $\gamma_{\Omega}^{k}$ of $\tau: \check{\Omega} \hookrightarrow \check{D}_{\Omega}$ coincides with the the $k$-th fundamental form $\psi_{\Omega}^{k}$ of $\sigma: \check{\Omega} \hookrightarrow \mathbb{P} U_{\mathbb{C}}$.

Proof. The proof is definition chasing. Since both the Hodge bundles $\mathcal{F}_{\Omega}^{p}$ and the osculating filtration $\mathcal{T}_{\Omega}^{k}$ are homogeneous, and the maps $\sigma$ and $\tau$ are $G_{\mathbb{C}^{-}}$equivariant, it suffices to show that $\gamma_{\Omega, o}^{k}=\psi_{\Omega, o}^{k}$ at the point $o=P / P \in \check{\Omega}$. The former is computed in $\S 3.3$; so it suffices to compute the latter and show that $\psi_{\Omega, o}^{k}$ agrees with (3.19). This follows directly from the the definition $\sigma(g P)=g \cdot \ell$ and the identifications (3.12) and (3.15).

Remark 4.5. A more detailed discussion of the fundamental forms of compact Hermitian symmetric spaces ( $\operatorname{such}$ as $\check{\Omega}$ ) may be found in $[10, \S 3]$

Corollary 4.6. The Hodge filtration $\left.\mathcal{F}_{\Omega}^{p}\right|_{\tau(\check{\Omega})}$ agrees with the osculating filtration $\mathcal{T}_{\sigma(\tilde{\Omega})}^{n-p}$. 


\subsection{Characteristic versus fundamental forms.}

Lemma 4.7. Let $f: M \hookrightarrow \check{D}$ be a $C Y$ map (§3.1). Let $\pi: \check{D} \rightarrow \mathbb{P} V_{\mathbb{C}}$ be the projection of §3.1. Then $\mathcal{T}_{\pi \circ f, x}^{n-k} \subset \mathcal{F}_{f, x}^{k}$ for all $x \in M$.

Proof. This follows directly from the definitions of horizontality ( 11.2$)$ and CalabiYau type (§3.1), and the osculating filtration (§4.1).

Remark 4.8. Let $f: M \hookrightarrow \check{D}$ be a CY map, and recall the projection $\pi: \check{D} \rightarrow \mathbb{P} V_{\mathbb{C}}$ of $\S 3.1$. By definition $f(x)=\mathcal{F}_{f, x}^{\bullet}$. So, if the Hodge and osculating filtrations agree, $\mathcal{F}_{f, x}^{k}=\mathcal{T}_{\pi \circ f, x}^{n-k}$, then we can recover $f$ from $\pi \circ f$.

Lemma 4.9. Let $f: M \hookrightarrow \check{D}$ be a $C Y$ map. If $\mathcal{T}_{\pi \circ f, x}^{n-k}=\mathcal{F}_{f, x}^{k}$ for all $x \in M$, then the characteristic and fundamental forms agree, $\mathbf{C}_{f}^{k}=\mathbf{F}_{f}^{n-k}$.

Proof. Again this is an immediate consequence of the definitions of the characteristic and fundamental forms ( $\S 2$ and $\S 4.2$, respectively).

Lemma 4.10. Let $f: M \hookrightarrow \check{D}_{\Omega}$ be a $C Y$ map. Suppose that the characteristic forms $\mathbf{C}_{f}^{\bullet}$ of $f$ are isomorphic to the characteristic forms $\mathbf{C}_{\Omega}^{\bullet}$ of $\tau: \check{\Omega} \hookrightarrow \check{D}_{\Omega}$. Then the fundamental forms $\mathbf{F}_{\pi \circ f}^{\bullet}$ and $\mathbf{F}_{\sigma}^{\bullet}$ are isomorphic.

Proof. The lemma is a corollary of Corollary 4.6 and Lemma 4.9.

Proof of Theorem 3.10. First observe that we may reduce to the case that $\check{\Omega}$ is irreducible: for if $\check{\Omega}$ factors as $\check{\Omega}_{1} \times \check{\Omega}_{2}$, then we have corresponding factorizations $\check{D}_{\Omega}=\check{D}_{\Omega_{1}} \times \check{D}_{\Omega_{2}}$ and $f=f_{1} \times f_{2}$ with $f_{i}: M \rightarrow \check{D}_{\Omega_{i}}$; the theorem holds for $f$ if and only if it holds for the $f_{i}$.

Now suppose that $\check{\Omega}$ is a projective space. Then $\check{\Omega}=\mathbb{P}^{1}$. In this case $\check{\Omega}=\check{D}_{\Omega}$, and the theorem is trivial. Likewise if $\check{\Omega}$ is a quadric hypersurface, then $\check{\Omega}=\check{D}_{\Omega}$, and the theorem is trivial. (In both these cases $\tau=\sigma$ and $\pi$ is the identity.)

The remainder of the theorem is essentially a corollary of Theorem 4.3 and Lemma 4.10. These results imply that there exists $g \in \operatorname{Aut}\left(U_{\mathbb{C}}\right)$ so that $g \circ \pi \circ f(M)$ is an open subset of $\pi \circ \tau(\check{\Omega})=\sigma(\check{\Omega})$. From Remark 4.8 we deduce that $g \circ f(M)$ is an open subset of $\tau(\check{\Omega})$. 


\section{Main Theorem 2}

In this section we give a precise statement (Theorem 5.14) and proof of Main Theorem 2. The theorem assumes a stronger form of isomorphism between the characteristic forms of $\tau$ and $f$ than Main Theorem 1 ; specifically the identification $\mathbf{F}_{\Omega} \simeq \mathbf{F}_{f}$ will respect the polarization $Q$ in a way that is made precise by working on a natural frame bundle $\mathcal{E}_{Q} \rightarrow \check{D}_{\Omega}$.

5.1. The frame bundle $\mathcal{E}_{Q} \rightarrow \check{D}_{\Omega}$. Let $d+1=\operatorname{dim} U_{\mathbb{C}}$, and let

$$
d^{p}+1:=\operatorname{dim} F^{p}
$$

be the dimensions of the flags $\left(F^{p}\right)$ parameterized by $\check{D}_{\Omega}$. Let $\mathcal{E}_{Q}$ be the set of all bases $\mathbf{e}=\left\{e_{0}, \ldots, e_{d}\right\}$ of $U_{\mathbb{C}}$ so that $Q\left(e_{j}, e_{k}\right)=\delta_{j+k}^{d}$. Note that we have bundle map

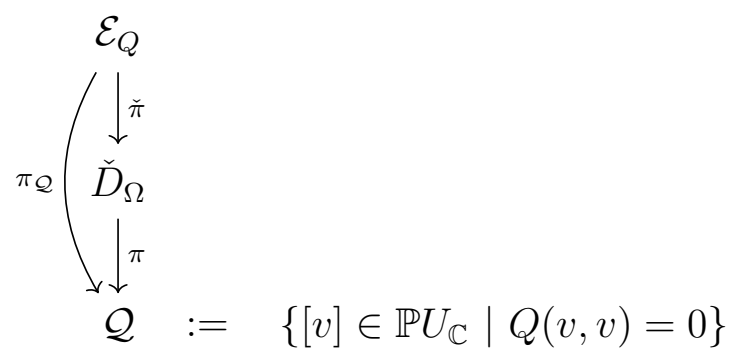

given by

$$
\begin{aligned}
\check{\pi}(\mathbf{e}) & =\left(F^{p}\right), \quad F^{p}=\operatorname{span}\left\{e_{0}, \ldots, e_{d^{p}}\right\} \\
\pi_{\mathcal{Q}}(\mathbf{e}) & =\left[e_{0}\right] .
\end{aligned}
$$

5.2. Maurer-Cartan form. The frame bundle $\mathcal{E}_{Q}$ is naturally identified with the Lie group $\operatorname{Aut}\left(U_{\mathbb{C}}, Q\right)$,

$$
\mathcal{E}_{Q} \simeq \operatorname{Aut}\left(U_{\mathbb{C}}, Q\right)
$$

and the bundle maps are equivariant with respect to the natural (left) action of $\operatorname{Aut}\left(U_{\mathbb{C}}, Q\right)$. Consequently, the (left-invariant) Maurer-Cartan form on $\operatorname{Aut}\left(U_{\mathbb{C}}, Q\right)$ defines a $\operatorname{Aut}\left(U_{\mathbb{C}}, Q\right)$-invariant coframing $\theta=\left(\theta_{j}^{k}\right) \in \Omega^{1}\left(\mathcal{E}_{Q}, \operatorname{End}\left(U_{\mathbb{C}}, Q\right)\right)$. Letting $e_{j}$ denote the natural map $\mathcal{E}_{Q} \rightarrow U_{\mathbb{C}}$, the coframing is determined by

$$
\mathrm{d} e_{j}=\theta_{j}^{k} e_{k}
$$


(The 'Einstein summation convention' is in effect throughout: if an index appears as both a subscript and a superscript, then it is summed over. For example, the righthand side of (5.2) should be read as $\sum_{k} \theta_{j}^{k} e_{k}$.) The form $\theta$ can be used to characterize horizontal maps as follows: let $f: M \rightarrow \check{D}_{\Omega}$ be any holomorphic map and define

$$
\mathcal{E}_{f}:=f^{*}\left(\mathcal{E}_{Q}\right) .
$$

In a mild abuse of notation, we let $\theta$ denote both the Maurer-Cartan form on $\mathcal{E}_{Q}$, and its pull-back to $\mathcal{E}_{f}$. Then it follows from the definition (2.2) that

$$
\begin{aligned}
& \text { the map } f \text { is horizontal if and only if }\left.\theta_{\nu}^{\mu}\right|_{\mathcal{E}_{f}}=0 \text { for all } \\
& d^{q+1}+1 \leq \mu \leq d^{q} \text { and } d^{p+1}+1 \leq \nu \leq d^{p} \text { with } p-q \geq 2 .
\end{aligned}
$$

5.3. Precise statement of Main Theorem 2. The precise statement (Theorem 5.14) of Main Theorem 2 is in terms of a decomposition of the Lie algebra $\operatorname{End}\left(U_{\mathbb{C}}, Q\right)$. Recall the Hodge decomposition (3.4), and define

$$
E_{\ell}:=\left\{\xi \in \operatorname{End}\left(U_{\mathbb{C}}, Q\right) \mid \xi\left(U^{p, q}\right) \subset U^{p+\ell, q-\ell}\right\} .
$$

Then

$$
\operatorname{End}\left(U_{\mathbb{C}}, Q\right)=\bigoplus_{\ell} E_{\ell}
$$

and this direct sum is a graded decomposition in the sense that the Lie bracket satisfies

$$
\left[E_{k}, E_{\ell}\right] \subset E_{k+\ell} .
$$

Let $\theta_{\ell} \in \Omega^{1}\left(\mathcal{E}_{Q}, E_{\ell}\right)$ denote the component of $\theta$ taking value in $E_{\ell}$. It follows from (5.3) that

$$
\text { a holomorphic map } f: M \rightarrow \check{D}_{\Omega} \text { is horizontal }
$$

$$
\text { if and only if }\left.\theta_{-\ell}\right|_{\mathcal{E}_{f}}=0 \text { for all } \ell \geq 2 \text {. }
$$

Let $\tilde{P} \subset \operatorname{Aut}\left(U_{\mathbb{C}}, Q\right)$ be the stabilizer of $\varphi=\tau(o) \in \check{D}$. Notice that the fibre $\check{\pi}^{-1}(\varphi) \subset \mathcal{E}_{Q}$ is isomorphic to $\tilde{P}$, and $\check{\pi}: \mathcal{E}_{Q} \rightarrow \check{D}_{\Omega}$ is a principle $\tilde{P}$-bundle. The Lie algebra of $\tilde{P}$ is

$$
E_{\geq 0}:=\bigoplus_{\ell \geq 0} E_{\ell} .
$$


Consequently, if $\theta=\theta_{\geq 0}+\theta_{-}$is the decomposition of $\theta$ into the components taking value in $E_{\geq 0}$ and $E_{-}:=\oplus_{\ell>0} E_{-\ell}$, respectively, then

$$
\operatorname{ker} \check{\pi}_{*}=\operatorname{ker} \theta_{\geq 0} \subset T \mathcal{E}_{Q} .
$$

We may further refine the decomposition (5.4) by taking the representation (3.5) into account. The latter allows us to view $\operatorname{End}\left(U_{\mathbb{C}}, Q\right)$ as a $G_{\mathbb{C}}-$ module via the adjoint action of $\operatorname{Aut}\left(U_{\mathbb{C}}, Q\right)$ on the endomorphism algebra. Likewise, we may regard $\mathfrak{g}_{\mathbb{C}}$ as a subalgebra of $\operatorname{End}\left(U_{\mathbb{C}}, Q\right)$ via the induced representation $\mathfrak{g} \hookrightarrow \operatorname{End}(U, Q)$. Since $\mathfrak{g}_{\mathbb{C}} \subset \operatorname{End}\left(U_{\mathbb{C}}, Q\right)$ is a $G_{\mathbb{C}^{-}}$-submodule and $G_{\mathbb{C}}$ is reductive, there exists a $G_{\mathbb{C}^{-}}$module decomposition

$$
\operatorname{End}\left(U_{\mathbb{C}}, Q\right)=\mathfrak{g}_{\mathbb{C}} \oplus \mathfrak{g}_{\mathbb{C}}^{\perp}
$$

Note that

$$
\left[\mathfrak{g}_{\mathbb{C}}, \mathfrak{g}_{\mathbb{C}}\right] \subset \mathfrak{g}_{\mathbb{C}} \text { and }\left[\mathfrak{g}_{\mathbb{C}}, \mathfrak{g}_{\mathbb{C}}^{\perp}\right] \subset \mathfrak{g}_{\mathbb{C}}^{\perp}
$$

where the Lie bracket is taken in $\operatorname{End}\left(U_{\mathbb{C}}, Q\right)$.

Both $\mathfrak{g}_{\mathbb{C}}$ and $\mathfrak{g}_{\mathbb{C}}^{\perp}$ inherit graded decompositions

$$
\mathfrak{g}_{\mathbb{C}}=\oplus \mathfrak{g}_{\ell} \quad \text { and } \quad \mathfrak{g}_{\mathbb{C}}^{\perp}=\oplus \mathfrak{g}_{\ell}^{\perp}
$$

defined by $\mathfrak{g}_{\ell}:=\mathfrak{g}_{\mathbb{C}} \cap E_{\ell}$ and $\mathfrak{g}_{\ell}^{\perp}:=\mathfrak{g}_{\mathbb{C}}^{\perp} \cap E_{\ell}$. From (5.5) and (5.8) we deduce

$$
\left[\mathfrak{g}_{k}, \mathfrak{g}_{\ell}\right] \subset \mathfrak{g}_{k+\ell} \text { and }\left[\mathfrak{g}_{k}, \mathfrak{g}_{\ell}^{\perp}\right] \subset \mathfrak{g}_{k+\ell}^{\perp} .
$$

Recall the Hodge decomposition (3.14) and note that $\mathfrak{g}_{\ell}=\mathfrak{g}_{\varphi}^{\ell,-\ell}$; in particular, $\mathfrak{g}_{\ell}=$ $\{0\}$ if $|\ell|>1$, so that

$$
\mathfrak{g}_{\mathbb{C}}=\mathfrak{g}_{1} \oplus \mathfrak{g}_{0} \oplus \mathfrak{g}_{-1}
$$

and

$$
\mathfrak{g}_{\ell}^{\perp}=E_{\ell} \text { for all }|\ell| \geq 2 .
$$

Set

$$
\begin{aligned}
\mathfrak{g}_{\geq 0} & =\bigoplus_{\ell \geq 0} \mathfrak{g}_{\ell} \quad \text { and } \quad \mathfrak{g}_{-}=\bigoplus_{\ell<0} \mathfrak{g}_{\ell} \\
\mathfrak{g}_{\geq 0}^{\perp} & =\bigoplus_{\ell \geq 0} \mathfrak{g}_{\ell}^{\perp} \quad \text { and } \quad \mathfrak{g}_{-}^{\perp}=\bigoplus_{\ell<0} \mathfrak{g}_{\ell}^{\perp} .
\end{aligned}
$$


Let $\theta_{\mathfrak{g} \geq 0}, \theta_{\mathfrak{g}_{\geq 0}^{\perp}}$,

$$
\omega:=\theta_{\mathfrak{g}_{-}} \quad \text { and } \quad \eta:=\theta_{\mathfrak{g}_{-}}
$$

denote the components of $\theta$ taking value in $\mathfrak{g}_{\geq 0}, \mathfrak{g}_{\geq 0}^{\perp}, \mathfrak{g}_{-}$and $\mathfrak{g}_{-}^{\perp}$, respectively.

Given any complex submanifold $\mathcal{M} \subset \mathcal{E}_{Q}$, we say that the restriction $\left.\omega\right|_{\mathcal{M}}$ is nondegenerate if the linear map

$$
\omega: T_{\mathbf{e}} \mathcal{M} \rightarrow \mathfrak{g}_{-}
$$

is onto for all $\mathbf{e} \in \mathcal{M}$.

Example 5.13. Recall the horizontal, equivariant embedding $\tau: \check{\Omega} \rightarrow \check{D}_{\Omega}$. It follows from (5.7) and the fact that $\tau: \check{\Omega} \hookrightarrow \check{D}_{\Omega}$ is $G_{\mathbb{C}}$ equivariant that

$$
\left.\eta\right|_{\mathcal{E}_{\tau}}=0
$$

and $\left.\omega\right|_{\mathcal{E}_{\tau}}$ is nondegenerate.

Our second main theorem asserts that these two properties suffice to characterize $\tau: \check{\Omega} \rightarrow \check{D}_{\Omega}$ up to the action of $\operatorname{Aut}\left(U_{\mathbb{C}}, Q\right)$.

Theorem 5.14. Let $f: M \rightarrow \check{D}_{\Omega}$ be a horizontal map of Calabi-Yau type. There exists $g \in \operatorname{Aut}\left(U_{\mathbb{C}}, Q\right)$ so that $g \circ f(M)$ is an open subset of $\tau(\check{\Omega})$ if and only if $\eta$ vanishes on $\mathcal{E}_{f}$.

The theorem is proved in $\S 5.5$.

5.4. Relationship to characteristic forms. The purpose of this section is to describe the characteristic forms $\mathbf{C}_{f}^{k}$ when $\left.\eta\right|_{\mathcal{E}_{f}}=0$. The precise statement is given by Proposition 5.18. It will be convenient to fix the following index ranges

$$
d^{n-k+1}+1 \leq \mu_{k}, \nu_{k} \leq d^{n-k} \text { with } k \geq 1 .
$$

As we will see below, the indices $1 \leq \mu_{1}, \nu_{1} \leq d^{n-1}$ are distinguished, and we will use the notation

$$
1 \leq a, b \leq d^{n-1}
$$

for this range. We claim that the equations

$$
\eta_{0}^{a}=0 \quad \text { and } \quad \theta_{0}^{a}=\omega_{0}^{a}, \quad \text { for all } 1 \leq a \leq d^{n-1}
$$


hold on $\mathcal{E}_{Q}$. (Note that the first implies the second, and visa versa.) The way to see this is to observe that (i) $\left(\theta_{0}^{a}\right)_{a=1}^{d^{n-1}}$ is precisely the component of $\theta$ taking value in

$$
E_{-1} \cap \operatorname{Hom}\left(\mathcal{F}_{\varphi}^{n}, \mathcal{F}_{\varphi}^{n-1}\right) \simeq \operatorname{Hom}\left(\mathcal{F}_{\varphi}^{n}, \mathcal{F}_{\varphi}^{n-1} / \mathcal{F}_{\varphi}^{n}\right)
$$

and (ii) the fact that $\tau$ is Calabi-Yau implies that the projection

$$
T_{o} \check{\Omega} \simeq \mathfrak{g}_{-} \rightarrow E_{-1} \cap \operatorname{Hom}\left(\mathcal{F}_{\varphi}^{n}, \mathcal{F}_{\varphi}^{n-1} / \mathcal{F}^{n-1}\right)
$$

is an isomorphism. Therefore,

$$
E_{-1} \cap \operatorname{Hom}\left(\mathcal{F}_{\varphi}^{n}, \mathcal{F}_{\varphi}^{n-1}\right)=\mathfrak{g}_{-1} \cap \operatorname{Hom}\left(\mathcal{F}_{\varphi}^{n}, \mathcal{F}_{\varphi}^{n-1}\right) \simeq \mathfrak{g}_{-1}
$$

There are three important consequences of (5.16). First, we have

$$
\theta_{0}^{a}=\omega_{0}^{a}
$$

which forces

$$
\eta_{0}^{a}=0
$$

for all $1 \leq a \leq d^{n-1}$. Second, the fact that $\gamma_{f, x}$ is an isomorphism implies that $\left.\omega\right|_{\mathcal{E}_{f}}$ is nondegenerate. Third, from $\mathfrak{g}_{-}=\mathfrak{g}_{-1}$ we conclude that

$$
\left(\theta_{\mathfrak{g}}\right)_{\nu_{\ell}}^{\mu_{k}}=0 \text { when } k-\ell \geq 2
$$

It follows from (5.16) that the remaining components of $\omega=\theta_{\mathfrak{g}_{-}}$may be expressed as

$$
\omega_{\nu_{k-1}}^{\mu_{k}}=r_{\nu_{k-1} a}^{\mu_{k}} \omega_{0}^{a}
$$

$k \geq 2$, for some holomorphic functions

$$
r_{\nu_{k-1} a}^{\mu_{k}}: \mathcal{E}_{Q} \rightarrow \mathbb{C}
$$

It will be convenient to extend the definition of $r_{\nu_{k-1} a}^{\mu_{k}}$ to $k=1$ by setting $r_{0 b}^{a}:=\delta_{b}^{a}$.

Proposition 5.18. Let $f: M \rightarrow \check{D}_{\Omega}$ be a horizontal map of Calabi-Yau type. Fix $\ell \geq 0$. The component of $\theta$ taking value in

$$
\mathfrak{g}_{-1}^{\perp} \bigcap \bigoplus_{k \leq \ell} \operatorname{Hom}\left(\mathcal{F}^{n-k+1}, \mathcal{F}^{n-k}\right)
$$

vanishes on $\mathcal{E}_{f}$ if and only if the

$$
\tilde{r}_{a_{k} \cdots a_{2} a_{1}}^{\mu_{k}}:=r_{\nu_{k-1} a_{k}}^{\mu_{k}} r_{\sigma_{k-2} a_{k-1}}^{\nu_{k-1}} \cdots r_{a_{2} a_{1}}^{\tau_{2}}
$$


are the coefficients of $\gamma_{f}^{k}$ for all $k \leq \ell$; that is,

$$
\gamma_{f, x}^{k}\left(\xi_{k}, \ldots, \xi_{1}\right)=\left\{e_{0} \mapsto \tilde{r}_{a_{k} \cdots a_{1}}^{\mu_{k}} \omega_{0}^{a_{k}}\left(\zeta_{k}\right) \cdots \omega_{0}^{a_{1}}\left(\zeta_{1}\right) e_{\mu_{k}} \bmod \mathcal{F}_{f, x}^{n-k+1}\right\}
$$

where $\zeta_{i} \in T_{\mathbf{e}} \mathcal{E}_{f}^{\prime}$ with $\mathbf{e}=\left\{e_{0}, \ldots, e_{d}\right\} \in \check{\pi}^{-1}(f(x))$ and $\check{\pi}_{*}\left(\zeta_{i}\right)=f_{*}\left(\xi_{i}\right)$. In particular, $\left.\eta\right|_{\mathcal{E}_{f}}=0$ if and only if the characteristic forms are given by (5.20) for all $k$.

Note that the component of $\theta$ taking value in (5.19) is $\left(\eta_{\nu_{\ell-1}}^{\mu_{\ell}}\right)_{\ell \leq k}$. The proposition is proved by induction in $§ \S 5.4 .1-5.4 .4$; because the first nontrivial step in the induction is $\ell=3$, we work through the cases $\ell=1,2,3$ explicitly.

Remark 5.21. Suppose that $\mathbf{e}=\left\{e_{0}, \ldots, e_{d}\right\} \in \mathcal{E}_{\tau, o}$. Making use of (5.16), we may identify $\left\{e_{1}, \ldots, e_{d^{n-1}}\right\}$ with a basis of $\left\{\xi_{1}, \ldots, \xi_{d^{n-1}}\right\}$ of $\mathfrak{g}_{-}$. Then the coefficients $r_{\nu_{k-1} a}^{\mu_{k}}$ are determined by

$$
\xi_{a}\left(e_{\nu_{k-1}}\right)=r_{\nu_{k-1} a}^{\mu_{k}} e_{\mu_{k}} \bmod \mathcal{F}_{\tau, o}^{n-k+1}
$$

There are two important consequences of this expression:

(a) It follows from (3.19) that (5.20) holds for $f=\tau$.

(b) Equation (5.16) tells us that $\mathfrak{g}_{-1}$ is the graph over $E_{-1} \cap \operatorname{Hom}\left(\mathcal{F}_{\varphi}^{n}, \mathcal{F}_{\varphi}^{n-1} / \mathcal{F}_{\varphi}^{n}\right)$ of a linear function

$$
R: E_{-1} \cap \operatorname{Hom}\left(\mathcal{F}_{\varphi}^{n}, \mathcal{F}_{\varphi}^{n-1} / \mathcal{F}_{\varphi}^{n}\right) \rightarrow \bigoplus_{k \geq 1} \operatorname{Hom}\left(\mathcal{F}_{\varphi}^{n-k}, \mathcal{F}_{\varphi}^{n-k-1} / \mathcal{F}_{\varphi}^{n-k}\right)
$$

The functions $r_{\nu_{k-1} a}^{\mu_{k}}(\mathbf{e})$ of (5.17) are the coefficients of this linear map with respect to the bases of $E_{-1} \cap \operatorname{Hom}\left(\mathcal{F}_{\varphi}^{n}, \mathcal{F}_{\varphi}^{n-1} / \mathcal{F}_{\varphi}^{n}\right)$ and $\oplus_{k \geq 1} \operatorname{Hom}\left(\mathcal{F}_{\varphi}^{n-k}, \mathcal{F}_{\varphi}^{n-k-1} / \mathcal{F}_{\varphi}^{n-k}\right)$ determined by $\mathbf{e} \in \mathcal{E}_{Q}$. Assuming that (5.20) holds, this implies that the $k$-th characteristic form of $f$ is isomorphic to that of $\tau$ in the following sense: given $\mathbf{e}_{o} \in \mathcal{E}_{\tau}$ in the fibre over $o$ and $\mathbf{e}_{x} \in \mathcal{E}_{f}$ in the fibre over $x$, there exists a unique $g \in \operatorname{Aut}\left(U_{\mathbb{C}}, Q\right) \simeq \mathcal{E}_{Q}$ so that $\mathbf{e}_{x}=g \cdot \mathbf{e}_{o}$. The group element $g$ defines an explicit isomorphism between $\operatorname{Sym}^{k} T_{o}^{*} \check{\Omega} \otimes \operatorname{Hom}\left(\mathcal{F}_{\tau, o}^{n}, \mathcal{F}_{\tau, o}^{n-k} / \mathcal{F}_{\tau, o}^{n-k+1}\right)$ and $\operatorname{Sym}^{k} T_{x}^{*} M \otimes \operatorname{Hom}\left(\mathcal{F}_{f, x}^{n}, \mathcal{F}_{f, x}^{n-k} / \mathcal{F}_{f, x}^{n-k+1}\right)$ that identifies the $k$-th characteristic forms $\gamma_{\tau, o}^{k}$ and $\gamma_{f, x}^{k}$ at $o$ and $x$, respectively. This is the precise sense in which the vanishing of $\eta$ on $\mathcal{E}_{f}$ is a refined notion of agreement of the characteristic forms.

Remark 5.23. Recalling (3.16), and the identification $U^{p, q}=\mathcal{F}_{\tau, o}^{p} / \mathcal{F}_{\tau, o}^{p+1},(5.22)$ implies that the system $\left\{r_{\nu_{k-1} a}^{\mu_{k}} Y_{\mu_{k}}=0\right\}$ of $d^{n-1}\left(d^{k-1}-d^{k}\right)$ equations in the $d^{k}-d^{k+1}$ unknowns $\left\{Y_{\mu_{k}}\right\}$ has only the trivial solution $Y_{\mu_{k}}=0$. 
5.4.1. The first characteristic form. Let $f: M \rightarrow \check{D}_{\Omega}$ be any horizontal map of Calabi-Yau type. On the bundle $\mathcal{E}_{f},(5.3)$ and (5.15) yield

$$
\mathrm{d} e_{0}=\theta_{0}^{0} e_{0}+\sum_{a=1}^{d^{n-1}} \omega_{0}^{a} e_{a}
$$

Consequently, the first characteristic form $\gamma_{f, x}: T_{x} M \rightarrow \operatorname{Hom}\left(\mathcal{F}_{f, x}^{n}, \mathcal{F}_{f, x}^{n-1} / \mathcal{F}_{f, x}^{n}\right)$ is given by

$$
\gamma_{f, x}(\xi)=\left\{e_{0} \mapsto \sum_{a=1}^{d^{n-1}} \omega_{0}^{a}(\zeta) e_{a} \bmod e_{0}\right\},
$$

where $\zeta \in T_{\mathbf{e}} \mathcal{E}_{f}$ with $\mathbf{e}=\left\{e_{0}, \ldots, e_{d}\right\} \in \check{\pi}^{-1}(f(x))$ and $\check{\pi}_{*}(\zeta)=f_{*}(\xi)$.

This establishes Proposition 5.18 for the trivial case that $\ell=1$.

5.4.2. The second characteristic form. From (5.3) we see that

$$
\theta_{0}^{\mu_{2}}=0 \quad \text { on } \quad \mathcal{E}_{f}
$$

for all $d^{n-1}+1 \leq \mu_{2} \leq d^{n-2}$. The derivative of this expression is given by the Maurer-Cartan equation

$$
\mathrm{d} \theta=-\frac{1}{2}[\theta, \theta] ;^{3} \text { equivalently, } \mathrm{d} \theta_{k}^{j}=-\theta_{\ell}^{j} \wedge \theta_{k}^{\ell} .
$$

Differentiating (5.25) and applying (5.3) yields

$$
0=\mathrm{d} \theta_{0}^{\mu_{2}}=-\theta_{a}^{\mu_{2}} \wedge \omega_{0}^{a}
$$

on $\mathcal{E}_{f}$. Cartan's Lemma [11] asserts that there exist holomorphic functions

$$
q_{a b}^{\mu_{2}}=q_{b a}^{\mu_{2}}: \mathcal{E}_{f} \rightarrow \mathbb{C}
$$

so that

$$
\theta_{a}^{\mu_{2}}=q_{a b}^{\mu_{2}} \omega_{0}^{b}
$$

The $q_{a b}^{\mu_{2}}$ are the coefficients of the second characteristic form; specifically,

$$
\gamma_{f, x}^{2}\left(\xi_{1}, \xi_{2}\right)=\left\{e_{0} \mapsto q_{a b}^{\mu_{2}} \omega_{0}^{a}\left(\zeta_{1}\right) \omega_{0}^{b}\left(\zeta_{2}\right) e_{\mu_{2}} \bmod \quad \mathcal{F}_{f, x}^{n-1}\right\},
$$

where $\zeta_{i} \in T_{\mathbf{e}} \mathcal{E}_{f}^{\prime}$ with $\mathbf{e}=\left\{e_{0}, \ldots, e_{d}\right\} \in \check{\pi}^{-1}(f(x))$ and $\check{\pi}_{*}\left(\zeta_{i}\right)=f_{*}\left(\xi_{i}\right)$.

\footnotetext{
${ }^{3}$ Given two Lie algebra valued 1 -forms $\phi$ and $\psi$, the Lie algebra valued 2-form $[\phi, \psi]$ is defined by $[\phi, \psi](u, v):=\frac{1}{2}([\phi(u), \psi(v)]-[\phi(v), \psi(u)]$.
} 
Remark 5.29. From Example 5.13, (5.17) and (5.27) we see that $q_{a b}^{\mu_{2}}=r_{a b}^{\mu_{2}}$ on $\mathcal{E}_{\tau}$.

Returning to the bundle $\mathcal{E}_{f}$, notice that $\left(\eta_{a}^{\mu_{2}}\right)$ is precisely the component of $\theta$ taking value in

$$
\mathfrak{g}_{-1}^{\perp} \cap \operatorname{Hom}\left(\mathcal{F}_{\varphi}^{n-1}, \mathcal{F}_{\varphi}^{n-2}\right)
$$

Comparing (5.17) and (5.27), we see that this component vanishes if and only if $r_{a b}^{\mu_{2}}=q_{a b}^{\mu_{2}}$ on $\mathcal{E}_{f}$. Noting that $\tilde{r}_{a b}^{\mu_{2}}=r_{a b}^{\mu_{2}}$, this yields Proposition 5.18 for $\ell=2$.

5.4.3. The third characteristic form. From (5.3) we see that

$$
\theta_{a}^{\mu_{3}}=0 \quad \text { on } \quad \mathcal{E}_{f}
$$

for all $d^{n-2}+1 \leq \mu_{2} \leq d^{n-3}$. Applying (5.3), the Maurer-Cartan equation (5.26), and substituting (5.27), we compute

$$
0=-\mathrm{d} \theta_{a}^{\mu_{3}}=\theta_{\nu_{2}}^{\mu_{3}} \wedge \theta_{a}^{\nu_{2}}=\theta_{\nu_{2}}^{\mu_{3}} \wedge q_{a b}^{\nu_{2}} \omega_{0}^{b}
$$

Again Cartan's Lemma implies there exist holomorphic functions $q_{a b c}^{\nu_{3}}: \mathcal{E}_{f} \rightarrow \mathbb{C}$, fully symmetric in the subscripts $a, b, c$, so that

$$
q_{a b}^{\nu_{2}} \theta_{\nu_{2}}^{\mu_{3}}=q_{a b c}^{\mu_{3}} \omega_{0}^{c}
$$

These functions are the coefficients of the third characteristic form of $f$ in the sense that

$$
\gamma_{f, x}^{3}\left(\xi_{1}, \xi_{2}, \xi_{3}\right)=\left\{e_{0} \mapsto q_{a b c}^{\mu_{3}} \omega_{0}^{a}\left(\zeta_{1}\right) \omega_{0}^{b}\left(\zeta_{2}\right) \omega_{0}^{c}\left(\zeta_{3}\right) e_{\mu_{3}} \bmod \quad \mathcal{F}_{f, x}^{n-2}\right\}
$$

where $\zeta_{i} \in T_{\mathbf{e}} \mathcal{E}_{f}^{\prime}$ with $\mathbf{e}=\left\{e_{0}, \ldots, e_{d}\right\} \in \check{\pi}^{-1}(f(x))$ and $\check{\pi}_{*}\left(\zeta_{i}\right)=f_{*}\left(\xi_{i}\right)$.

To prove Proposition 5.18 for $\ell=3$, note that $§ 5.4 .2$ yields $q_{a b}^{\mu_{2}}=r_{a b}^{\mu_{2}}$. Then we can solve (5.31) for $\theta_{\nu_{2}}^{\mu_{3}}$ (Remark 5.23). In particular, there exist $q_{\nu_{2} a}^{\mu_{3}}$ so that $\theta_{\nu_{2}}^{\mu_{3}}=q_{\nu_{2} a}^{\mu_{3}} \omega_{0}^{a}$. The component of $\theta$ taking value in

$$
\mathfrak{g}_{-1}^{\perp} \cap \operatorname{Hom}\left(\mathcal{F}_{\varphi}^{n-2}, \mathcal{F}_{\varphi}^{n-3}\right)
$$

vanishes (equivalently, $\eta_{\nu_{2}}^{\mu_{3}}=0$ ) if and only if these $q_{\nu_{2} a}^{\mu_{3}}$ are the $r_{\nu_{2} a}^{\mu_{3}}$ of (5.17); equivalently, (5.20) holds for $k=3$. This is Proposition 5.18 for $\ell=3$. 
5.4.4. And so on. Assume that Proposition 5.18 holds for a fixed $\ell \geq 3$. Then we have $\theta_{\nu_{k-1}}^{\mu_{k}}=\omega_{\nu_{k-1}}^{\mu_{k}}=r_{\nu_{k-1} a}^{\mu_{k}} \omega_{0}^{a}$ for all $k \leq \ell$. As in $\S \S 5.4 .2-5.4 .3$ we obtain the coefficients of the $(\ell+1)$-st characteristic form by differentiating $\theta_{\nu_{\ell-1}}^{\mu_{\ell+1}}=0$ and invoking Cartan's Lemma to obtain

$$
r_{\nu_{\ell-1} a}^{\sigma_{\ell}} \theta_{\sigma_{\ell}}^{\mu_{\ell+1}}=q_{\nu_{\ell-1} a b}^{\mu_{\ell+1}} \omega_{0}^{b}
$$

for some holomorphic functions $q_{\nu_{\ell-1} a b}^{\mu_{\ell+1}}: \mathcal{E}_{f} \rightarrow \mathbb{C}$, symmetric in $a, b$. Then Remark 5.23 implies that there exist $q_{\nu_{\ell} a}^{\mu_{\ell+1}}: \mathcal{E}_{f} \rightarrow \mathbb{C}$ so that

$$
\theta_{\nu_{\ell}}^{\mu_{\ell+1}}=q_{\nu_{\ell} a}^{\mu_{\ell+1}} \omega_{0}^{a}
$$

The $q_{a_{\ell} \cdots a_{1} a_{0}}^{\mu_{\ell+1}}:=q_{\nu_{\ell} a_{\ell}}^{\mu_{\ell+1}} r_{\sigma_{\ell-1} a_{\ell-1}}^{\nu_{\ell}} \cdots r_{a_{1} a_{0}}^{\tau_{2}}$ are the coefficients of the $(\ell+1)$-st characteristic form of $f$ in the sense that

$$
\gamma_{f, x}^{\ell+1}\left(\xi_{\ell}, \ldots, \xi_{0}\right)=\left\{e_{0} \mapsto q_{a_{\ell} \cdots a_{0}}^{\mu_{\ell+1}} \omega_{0}^{a_{\ell}}\left(\zeta_{k}\right) \cdots \omega_{0}^{a_{0}}\left(\zeta_{0}\right) e_{\mu_{\ell+1}} \bmod \mathcal{F}_{f, x}^{n-\ell}\right\}
$$

where $\zeta_{i} \in T_{\mathbf{e}} \mathcal{E}_{f}^{\prime}$ with $\mathbf{e}=\left\{e_{0}, \ldots, e_{d}\right\} \in \check{\pi}^{-1}(f(x))$ and $\check{\pi}_{*}\left(\zeta_{i}\right)=f_{*}\left(\xi_{i}\right)$. The component of $\theta$ taking value in

$$
\mathfrak{g}_{-1}^{\perp} \cap \operatorname{Hom}\left(\mathcal{F}_{\varphi}^{n-\ell}, \mathcal{F}_{\varphi}^{n-\ell-1}\right)
$$

vanishes (equivalently, $\eta_{\nu_{\ell}}^{\mu_{\ell+1}}=0$ ), if and only if the $q_{\nu_{\ell} a}^{\mu_{\ell+1}}$ are the $r_{\nu_{\ell} a}^{\mu_{\ell+1}}$ of (5.17); equivalently, (5.20) holds for $k \leq \ell+1$.

This establishes Proposition 5.18.

\subsection{Proof of Theorem 5.14.}

Claim 5.34. It suffices to show that $\mathcal{E}_{f}$ admits a sub-bundle $\mathcal{E}_{f}^{\prime}$ on which $\theta_{\mathfrak{g}^{\perp}}$ vanishes.

Example 5.35 (Subbundle $\mathcal{G} \subset \mathcal{E}_{\tau}$ ). The bundle $\mathcal{E}_{\tau} \rightarrow \check{\Omega}$ admits a subbundle $\mathcal{G}$ that is isomorphic to the image of $G_{\mathbb{C}}$ in $\operatorname{Aut}\left(U_{\mathbb{C}}, Q\right)$, and on which the entire component $\theta_{\mathfrak{g}^{\perp}}$ of $\theta$ taking value in $\mathfrak{g}^{\perp}$ vanishes. To see this, fix a basis $\mathbf{e}_{o}=\left\{e_{0}, \ldots, e_{d}\right\}$ that is adapted to the Hodge decomposition (3.4) in the sense that $e_{0}$ spans $U^{n, 0},\left\{e_{1}, \ldots, e_{d_{1}}\right\}$ spans $U^{n-1,1}$, et cetera, so that $\left\{e_{d_{q-1}+1}, \ldots, e_{d_{q}}\right\}$ spans $U^{n-q, q}$, for all $q$. Then $\mathbf{e}_{o} \in \mathcal{E}_{\tau}$, and

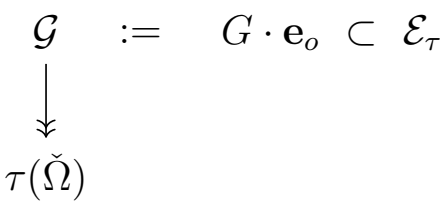


is a $G_{\mathbb{C}^{-}}$-homogenous subbundle with the properties that

$$
\left.\theta_{\mathfrak{g}^{\perp}}\right|_{\mathcal{G}}=0
$$

(in particular, $\left.\eta\right|_{\mathcal{G}}=0$ ) and $\left.\theta_{\mathfrak{g}}\right|_{\mathcal{G}}$ is a coframing of $\mathcal{G}$ (so that $\left.\omega\right|_{\mathcal{G}}$ is nondegenerate). Proof. Recalling (5.8), the Maurer-Cartan equation $\mathrm{d} \theta=-\frac{1}{2}[\theta, \theta]$ implies that $\left\{\theta_{\mathfrak{g}^{\perp}}=\right.$ $0\}$ is a Frobenius system on $\mathcal{E}_{Q}$. Notice that the bundle $\mathcal{G} \subset \mathcal{E}_{Q}$ of Example 5.35 is the maximal integral through $\mathbf{e}_{o}$. Since $\theta$ is $\operatorname{Aut}\left(U_{\mathbb{C}}, Q\right)$-invariant, it follows that the maximal integral manifolds of the Frobenius system are the $g \cdot \mathcal{G}$, with $g \in \operatorname{Aut}\left(U_{\mathbb{C}}, Q\right)$. Therefore, $g \cdot \mathcal{E}_{f}^{\prime} \subset \mathcal{G}$ for some $g \in \operatorname{Aut}\left(U_{\mathbb{C}}, Q\right)$. From the $\operatorname{Aut}\left(U_{\mathbb{C}}, Q\right)$-equivariance of $\check{\pi}$ we conclude that $g \circ f(M) \subset \check{\Omega}$.

We will show that $\mathcal{E}_{f}$ admits a sub-bundle $\mathcal{E}_{f}^{\prime}$ on which $\theta_{\mathfrak{g}^{\perp}}$ vanishes by induction. Given $\ell \geq-1$, suppose that $\mathcal{E}_{f}$ admits a subbundle $\mathcal{E}_{f}^{\ell}$ on which the form $\theta_{\mathfrak{g}_{k}^{\perp}}$ vanishes for all $k \leq \ell$. This inductive hypothesis holds for $\ell=-1$ with $\mathcal{E}_{f}=\mathcal{E}_{f}^{-1}$.

Claim 5.37. A maximal such $\mathcal{E}_{f}^{\ell}$ will have the property that the linear map

$$
\theta_{\geq \ell+2}: \operatorname{ker} \omega \subset T_{\mathrm{e}} \mathcal{E}_{f}^{\ell} \rightarrow E_{\geq \ell+2}
$$

is onto for all $\mathbf{e} \in \mathcal{E}_{f}^{\ell}$.

Proof. Recollect that $\mathcal{E}_{Q} \rightarrow \check{D}_{\Omega}$ is a principal $\tilde{P}$-bundle. Given $g \in \tilde{P}$, let

$$
R_{g}: \mathcal{E}_{Q} \rightarrow \mathcal{E}_{Q}
$$

denote the right action of $\tilde{P}$. Set $\tilde{P}_{\ell+2}:=\exp \left(E_{\geq \ell+2}\right) \subset \tilde{P}$. Then

$$
\tilde{\mathcal{E}}_{f}^{\ell}:=\left\{R_{g} \mathbf{e} \mid g \in \tilde{P}_{\ell+2}, \mathbf{e} \in \mathcal{E}_{f}^{\ell}\right\} \supset \mathcal{E}_{f}^{\ell}
$$

is a bundle over $M$, and $\theta_{\geq \ell+2}: \operatorname{ker} \omega \subset T_{\mathbf{e}} \tilde{\mathcal{E}}_{f}^{\ell} \rightarrow E_{\geq \ell+2}$ onto by construction. Additionally, $R_{g}^{*} \theta=\operatorname{Ad}_{g^{-1}} \theta$ implies that $\theta_{\mathfrak{g}_{\leq \ell}^{\perp}}$ vanishes on $\tilde{\mathcal{E}}_{f}^{\ell}$.

Given $\mathcal{E}_{f}^{\ell}$, which we assume to be maximal, we will show that $\mathcal{E}_{f}^{\ell+1} \subset \mathcal{E}_{f}^{\ell}$ exists. This will complete the inductive argument establishing the existence of the bundle $\mathcal{E}_{f}^{\prime}$ in Claim 5.34.

Claim 5.38. There exists a holomorphic map $\lambda: \mathcal{E}_{f}^{\ell} \rightarrow \operatorname{Hom}\left(\mathfrak{g}_{-}, \mathfrak{g}_{\ell+1}^{\perp}\right)=\mathfrak{g}^{\perp} \otimes \mathfrak{g}_{-}^{*}$ so that

$$
\theta_{\mathfrak{g}_{\ell+1}^{\perp}}=\lambda(\omega)
$$


Proof. Since $\theta_{\mathfrak{g}_{\ell}^{\perp}}$ vanishes on $\mathcal{E}_{f}^{\ell}$, the exterior derivative $\mathrm{d} \theta_{\mathfrak{g}_{\ell}^{\perp}}$ must as well. Making use of the Maurer-Cartan equation (5.26) and the relations (5.10) we compute

$$
0=\mathrm{d} \theta_{\mathfrak{g}_{\ell}^{\perp}}=-\left[\theta_{\mathfrak{g}_{\ell+1}^{\perp}}, \omega\right]
$$

on $\mathcal{E}_{f}^{\ell}$. The claim will then follow from Cartan's Lemma [11, Lemma A.1.9] once we show that the natural map

$$
\mathfrak{g}_{\ell+1}^{\perp} \rightarrow \mathfrak{g}_{\ell}^{\perp} \otimes \mathfrak{g}_{-}^{*} \quad \text { is injective. }
$$

The map (5.45) fails to be injective if and only if

$$
\Gamma_{\ell+1}:=\left\{\zeta \in \mathfrak{g}_{\ell+1}^{\perp} \mid[\xi, \zeta]=0 \forall \xi \in \mathfrak{g}_{-}\right\}
$$

is nontrivial. The Jacobi identity implies that $\Gamma_{\ell+1}$ is a $\mathfrak{g}_{0}$-module. Inductively define $\Gamma_{m}:=\mathfrak{g}_{+}\left(\Gamma_{m-1}\right) \subset \mathfrak{g}_{m}^{\perp}$. The Jacobi identity again implies that $\Gamma=\oplus_{m \geq \ell+1} \Gamma_{m}$ is a $\mathfrak{g}_{\mathbb{C}}$-module.

Let $\mathrm{E} \in \operatorname{End}\left(U_{\mathbb{C}}, Q\right)$ be the endomorphism acting on $E_{m}$ by the scalar $m$. (That is, (5.4) is the eigenspace decomposition for E.) Then $E \subset \mathfrak{g}_{\mathbb{C}}$ lies in the center of $\mathfrak{g}_{0}=\mathfrak{k}_{\mathbb{C}}\left[1\right.$, Proposition 3.1.2]. As a nontrivial semisimple element of $\mathfrak{g}_{\mathbb{C}}, \mathrm{E}$ will act on any nontrivial $\mathfrak{g}_{\mathbb{C}}$-module by both positive and negative eigenvalues. Since $\ell \geq-1$, we see that $E$ acts on $\Gamma$ by only non-negative eigenvalues. This forces $\Gamma=\Gamma_{\ell+1}=\Gamma_{0}$ and $\left[\mathfrak{g}_{\mathbb{C}}, \Gamma\right]=0$.

A final application of the Jacobi identity implies that $\mathfrak{g}_{\mathbb{C}} \oplus \Gamma$ is a subalgebra of $\operatorname{End}\left(U_{\mathbb{C}}, Q\right)$. Since $\mathfrak{g}_{\mathbb{C}} \subset \operatorname{End}\left(U_{\mathbb{C}}, Q\right)$ is a maximal proper subalgebra [3, Theorem 1.5], and $\mathfrak{g}_{\mathbb{C}} \oplus \Gamma_{0} \neq \operatorname{End}\left(U_{\mathbb{C}}, Q\right)$, it follows that $\Gamma=\Gamma_{0}=0$.

So to complete our inductive argument establishing the existence of $\mathcal{E}_{f}^{\prime}$ it suffices to show that there exists a subbundle $\mathcal{E}_{f}^{\ell+1} \subset \mathcal{E}_{f}^{\ell}$ on which $\lambda$ vanishes.

Claim 5.42. The map $\lambda$ takes value in the kernel of the Lie algebra cohomology [12] differential

$$
\delta^{1}: \mathfrak{g}^{\perp} \otimes \mathfrak{g}_{-}^{*} \rightarrow \mathfrak{g}^{\perp} \otimes \bigwedge^{2} \mathfrak{g}_{-}^{*}
$$

defined by

$$
\delta^{1}(\alpha)\left(\xi_{1}, \xi_{2}\right):=\left[\alpha\left(\xi_{1}\right), \xi_{2}\right]-\left[\alpha\left(\xi_{2}\right), \xi_{1}\right],
$$

where $\alpha \in \mathfrak{g}^{\perp} \otimes \mathfrak{g}_{-}^{*}=\operatorname{Hom}\left(\mathfrak{g}_{-}, \mathfrak{g}^{\perp}\right)$ and $\xi_{i} \in \mathfrak{g}_{-}$. 
Proof. Substituting (5.39) into (5.40) yields $[\lambda(\omega), \omega]=0$. The claim follows.

Claim 5.43. Suppose $\lambda$ takes value in the image of the Lie algebra cohomology differential

$$
\delta^{0}: \mathfrak{g}^{\perp} \rightarrow \mathfrak{g}^{\perp} \otimes \mathfrak{g}_{-}^{*}
$$

defined by

$$
\delta^{0}(\zeta)(\xi):=[\xi, \zeta]
$$

with $\zeta \in \mathfrak{g}^{\perp}$ and $\xi \in \mathfrak{g}_{-}$. Then there exists a subbundle $\mathcal{E}_{f}^{\ell+1} \subset \mathcal{E}_{f}^{\ell}$ on which $\lambda$ vanishes.

Proof. Differentiating (5.39) yields

$$
0=\frac{1}{2} \sum_{a+b=\ell+1}\left[\theta_{a}, \theta_{b}\right]_{\mathfrak{g}^{\perp}}+\mathrm{d} \lambda \wedge \omega-\lambda\left(\left[\theta_{\mathfrak{g}_{0}}, \omega\right]\right)
$$

Claim 5.37 implies that $\theta(Z)=\zeta$ determines a unique, holomorphic vector field $Z$ on $\mathcal{E}_{f}^{\ell}$. (At the point $\mathbf{e} \in \mathcal{E}_{f}^{\ell}$, the vector field is given by $Z_{\mathbf{e}}=\left.\frac{\mathrm{d}}{\mathrm{d} t} R_{\exp (t \zeta)} \mathbf{e}\right|_{t=0}$.) Taking the interior product of $Z$ with (5.44) yields

$$
0=(Z \lambda)(\omega)+[\zeta, \omega]
$$

That is, $Z \lambda=\mathrm{d} \lambda(Z)=\operatorname{ad}_{\zeta}$. Given $\mathbf{e} \in \mathcal{E}_{f, x}^{\ell}$, set $\lambda_{t}:=\lambda_{\mathbf{e}(t)}$ with $\mathbf{e}(t):=R_{\exp (t \zeta)} \mathbf{e}$. Then (5.45) implies we may solve $\lambda_{t}=0$ for $t$ if and only if $\lambda_{\mathbf{e}}$ takes value in the image of $\delta^{0}$.

It follows from Claims 5.42 and 5.43 that the bundle $\mathcal{E}_{f}^{\ell+1}$ exists if the cohomology group

$$
H^{1}\left(\mathfrak{g}_{-}, \mathfrak{g}^{\perp}\right):=\frac{\operatorname{ker} \delta^{1}}{\operatorname{im} \delta^{0}}
$$

is trivial. In general $H^{1}\left(\mathfrak{g}_{-}, \mathfrak{g}^{\perp}\right) \neq 0$. Happily it happens that we don't need all of $H^{1}\left(\mathfrak{g}_{-}, \mathfrak{g}^{\perp}\right)$ to vanish, just the positively graded component. To be precise, the gradings (5.9) induce a graded decomposition

$$
\mathfrak{g}^{\perp} \otimes \mathfrak{g}_{-}^{*}=\bigoplus_{\ell} \mathfrak{g}_{\ell}^{\perp} \otimes \mathfrak{g}_{-}^{*}
$$


Since $\mathfrak{g}_{-}=\mathfrak{g}_{-1}$, the dual $\mathfrak{g}_{-}^{*}$ has graded degree 1 . Consequently, $\mathfrak{g}_{\ell}^{\perp} \otimes \mathfrak{g}_{-}^{*}$ has graded degree $\ell+1$. The Lie algebra cohomology differentials $\delta^{1}$ and $\delta^{0}$ preserve this bigrading, and so induce a graded decomposition of the cohomology

$$
H^{1}\left(\mathfrak{g}_{-}, \mathfrak{g}^{\perp}\right)=\bigoplus_{\ell} H_{\ell}^{1}
$$

where the component of graded degree $\ell+1$ is

$$
H_{\ell+1}^{1}:=\frac{\operatorname{ker}\left\{\delta^{1}: \mathfrak{g}_{\ell}^{\perp} \otimes \mathfrak{g}_{-}^{*} \rightarrow \mathfrak{g}_{\ell-1}^{\perp} \otimes \bigwedge^{2} \mathfrak{g}_{-}^{*}\right\}}{\operatorname{im}\left\{\delta^{0}: \mathfrak{g}_{\ell+1}^{\perp} \rightarrow \mathfrak{g}_{\ell}^{\perp} \rightarrow \mathfrak{g}_{-}^{*}\right\}}
$$

Since $\lambda$ takes value in $\mathfrak{g}_{\ell+1}^{\perp} \otimes \mathfrak{g}_{-}^{*}$, and the latter is of pure graded degree $\ell+2 \geq 1$. Consequently,

$$
\begin{gathered}
\text { there exists a subbundle } \mathcal{E}_{f}^{\prime} \text { of } \mathcal{E}_{f} \text { on which } \\
\theta_{\mathfrak{g}^{\perp}} \text { vanishes if } H_{m}^{1}=0 \text { for all } m \geq 1 .
\end{gathered}
$$

To complete the proof of Theorem 5.14 we make the following observations: First, as in the proof of Theorem 3.10 we may reduce to the case that $\check{\Omega}$ is irreducible. Also as in that proof, the case that $\check{\Omega}$ is either a projective space (necessarily $\mathbb{P}^{1}$ ) or a quadric hypersurface is trivial.

In the remaining cases $H_{m}^{1}=0$ for all $m \geq 1$; this is a consequence of Kostant's theorem [12] on Lie algebra cohomology; see [10, Proposition 7] or [13, §7.3]. The theorem now follows from Claim 5.34 and (5.46).

\section{REFERENCES}

[1] Andreas Čap and Jan Slovák. Parabolic geometries. I, volume 154 of Mathematical Surveys and Monographs. American Mathematical Society, Providence, RI, 2009. Background and general theory.

[2] E. B. Dynkin. Maximal subgroups of the classical groups. Trudy Moskov. Mat. Obšč., 1:39-166, 1952.

[3] E. B. Dynkin. The maximal subgroups of the classical groups. Amer. Math. Soc. Trans., 6:245378, 1957. Translation of [2].

[4] Ralf Gerkmann, Mao Sheng, Duco van Straten, and Kang Zuo. On the monodromy of the moduli space of Calabi-Yau threefolds coming from eight planes in $\mathbb{P}^{3}$. Math. Ann., 355(1):187-214, 2013. 
[5] Mark Green, Phillip Griffiths, and Matt Kerr. Néron models and boundary components for degenerations of Hodge structure of mirror quintic type. In Curves and abelian varieties, volume 465 of Contemp. Math., pages 71-145. Amer. Math. Soc., Providence, RI, 2008.

[6] Mark Green, Phillip Griffiths, and Matt Kerr. Mumford-Tate groups and domains: their geometry and arithmetic, volume 183 of Annals of Mathematics Studies. Princeton University Press, Princeton, NJ, 2012.

[7] Phillip A. Griffiths. Periods of integrals on algebraic manifolds. I. Construction and properties of the modular varieties. Amer. J. Math., 90:568-626, 1968.

[8] Phillip A. Griffiths. Periods of integrals on algebraic manifolds. II. Local study of the period mapping. Amer. J. Math., 90:805-865, 1968.

[9] Benedict H. Gross. A remark on tube domains. Math. Res. Lett., 1(1):1-9, 1994.

[10] Jun-Muk Hwang and Keizo Yamaguchi. Characterization of Hermitian symmetric spaces by fundamental forms. Duke Math. J., 120(3):621-634, 2003.

[11] Thomas A. Ivey and J. M. Landsberg. Cartan for beginners: differential geometry via moving frames and exterior differential systems, volume 61 of Graduate Studies in Mathematics. American Mathematical Society, Providence, RI, 2003.

[12] Bertram Kostant. Lie algebra cohomology and the generalized Borel-Weil theorem. Ann. of Math. (2), 74:329-387, 1961.

[13] Joseph M. Landsberg and Colleen Robles. Fubini-Griffiths-Harris rigidity and Lie algebra cohomology. Asian J. Math., 16(4):561-586, 2012.

[14] Keiji Matsumoto, Takeshi Sasaki, and Masaaki Yoshida. The monodromy of the period map of a 4-parameter family of $K 3$ surfaces and the hypergeometric function of type $(3,6)$. Internat. J. Math., 3(1):164, 1992.

[15] Takeshi Sasaki, Keizo Yamaguchi, and Masaaki Yoshida. On the rigidity of differential systems modelled on Hermitian symmetric spaces and disproofs of a conjecture concerning modular interpretations of configuration spaces. In CR-geometry and overdetermined systems (Osaka, 1994), volume 25 of Adv. Stud. Pure Math., pages 318-354. Math. Soc. Japan, Tokyo, 1997.

[16] Mao Sheng, Jinxing Xu, and Kang Zuo. The monodromy groups of Dolgachev's CY moduli spaces are Zariski dense. Adv. Math., 272:699-742, 2015.

[17] Mao Sheng and Kang Zuo. Polarized variation of Hodge structures of Calabi-Yau type and characteristic subvarieties over bounded symmetric domains. Math. Ann., 348(1):211-236, 2010. E-mail address: robles@math.duke.edu 